\title{
Inclusión de estándares de derechos humanos en el marco de regulación de la Universidad Nacional de Asunción desde la década del noventa
}

\section{Inclusion of human rights standards within the regulatory framework of the National University of Asunción since the nineties}

\author{
Elba Núñez ${ }^{a}$
}

\section{Resumen}

El presente artículo es la primera fase de un estudio cualitativo de revisión documental cuyo objetivo es contribuir a la descripción y análisis de las principales reformas legales que se han registrado en la Universidad Nacional de Asunción (UNA) desde la década del go e identificar el grado de inclusión de estándares de derechos humanos. Parte de una revisión de la educación universitaria como derecho humano, basándose en instrumentos internacionales y compromisos que asumió el Estado paraguayo en la materia, para luego dar paso a la exploración de los antecedentes que hablan de la inclusión de los estándares de derechos en los marcos legales aprobados en la UNA a partir de la década del ' 90 y algunos indicadores que muestran la brecha que persisten para su cumplimiento.

Palabras clave: inclusión, derechos humanos, desigualdad, educación universitaria.

\begin{abstract}
Abstrac
This article is the first phase of a qualitative documentary review study whose objective is to contribute to the description and analysis of the main legal reforms that have been registered in the National University of Asuncion (UNA) since the 1990s, and to identify the degree of inclusion of human rights standards. Part of a review of university education as a human right, based on international instruments and commitments assumed by the Paraguayan State in the matter, and then give way to the exploration of the antecedents that speak of the inclusion of the standards of rights in the legal framework approved by the UNA (National University of Asuncion) from the $1990 \mathrm{os}$ and some indicators that show the gap that persist for its fulfillment.
\end{abstract}

Keywords: inclusion, human rights, inequality, university education.

Kera Yvoty: reflexiones sobre la cuestión social. Vol. 1, 2016, 91-102.

ISSN (impreso): 2519-7797

a Universidad Nacional de Asunción, Instituto de Trabajo Social, Paraguay.

Correspondencia a: enunez@rieder.net.py

Cita:

Núñez, E. (2016). Inclusión de estándares de derechos humanos en el marco de regulación de la Universidad Nacional de Asunción desde la década del noventa. Kera Yvoty: reflexiones sobre la cuestión social, 1, 91-102.

Recibido:

13 octubre 2016

Aceptado:

29 noviembre 2016 


\section{Introducción}

El trabajo asume la estrecha relación existente entre educación, democracia, desarrollo y sociedad. Es la primera fase de un estudio cualitativo de revisión documental, que busca responder a la pregunta ¿Cuáles han sido los cambios normativos para las universidades en Paraguay desde la década del 90 y en qué medida han posibilitado la inclusión de estándares de derechos humanos en la Universidad Nacional de Asunción?

Se propone como objetivo contribuir a la descripción y análisis de las reformas legales en materia de educación superior desde la década del 90 e identificar el grado de inclusión de estándares de derechos humanos en el marco legal vigente en la Universidad Nacional de Asunción.

La propia Declaración Universal de los Derechos Humanos de 1948, establece un marco general del derecho a la educación estableciendo que "Toda persona tiene derecho a la educación. La instrucción técnica y profesional habrá de ser generalizada; el acceso a los estudios superiores será igual para todos...."

UNESCO (2007) define que

[...] la educación es un derecho humano que permite a las personas ejercer los otros derechos humanos. Por esta razón, nadie puede quedar excluido de ella. El derecho a la educación se ejerce en la medida que las personas, más allá de tener acceso a la escuela, puedan desarrollarse plenamente y continuar aprendiendo. (p. 7)

Paraguay en el Foro Mundial de Educación del año 2015 de la UNESCO, ratifica la Declaración de Incheón, donde se asume que "la educación es un bien público, un derecho humano fundamental y la base para garantizar la realización de otros derechos".

Encuantoalaautonomíauniversitaria, Zárate et al. (2008, p. 144) plantea que existen tres tipos de autonomía: a) autonomía administrativa, entendida como la capacidad de organizarse libremente y darse gobierno propio b) autonomía económica, el Estado debe subsidiar a la institución con recursos suficientes para su funcionamiento; y, c) autonomía docente, entendida fundamentalmente como la libertad de cátedra, de expresión y de pensamiento.

El hito histórico más importante a nivel universitario de reforma universitaria, ha sido la reforma de Córdoba de 1918 que al decir de Tünnermann (2008, pp. 17-18)

[...] trajo a las universidades
latinoamericanas la autonomía
universitaria, como su fruto más
preciado. En cuanto a la función
social, abogó por la elección de
autoridades universitarias por la
propia academia y cogobierno con
postulados que hoy están consagrados
en las leyes y estatutos universitarios
de la región.

En cuanto al desarrollo de las universidades públicas en América Latina y el Caribe, hasta la década de los 8o, la educación superior pública con autonomía institucional, tenía el predominio en la región sobre la universidad privada, tal como señala López (2008, p. 272)

[...] al final de los 8 os y los principios de los 9os, la globalización implicó para América Latina estrategias neoliberales. Estas estrategias de mercado tuvieron un impacto en la privatización creciente de la educación superior y en el deterioro de las universidades públicas debido a la carencia de financiación apropiada, entre otros factores.

Paraguay en comparación con los países de la región, se ubica tardíamente en la apertura de universidades, creando su primera universidad pública, la Universidad Nacional deAsunción (UNA) a fines del siglo XIX. La UNA fue la primera universidad del Paraguay y su marco normativo fue 
modificándose paulatinamente, de acuerdo al contexto, la influencia de grupos de interés y la incidencia de movimientos sociales, en especial de los estudiantes.

Rivarola (2003, p. 38) señala que la Universidad Nacional de Asunción fue creada durante la etapa denominada "de reconstrucción" y, durante el periodo dictatorial (1954-1989) se consolidó el control y la dependencia de la universidad del poder político.

El trabajo se organiza en los siguientes apartados: i) En el primero se aborda la conceptualización de la educación universitaria desde el marco de los derechos humanos ii) En el segundo se recupera la evolución de la inclusión de los derechos humanos en el marco legal nacional iii) En el tercero se aborda la autonomía universitaria y reformulación de la relación Estado - Universidad iv) En el cuarto se analiza la desigualdad en la educación universitaria pública v) En quinto seanaliza la participación estudiantil y demandas por la autonomía y democratización de la UNA $y$, vi) En el último apartado se presentan las conclusiones.

\section{Materiales y Métodos}

El estudio ha sido cualitativo, descriptivo, en una primera etapa consistente en una revisión documental de los cambios legales registrados en el país con relación a la educación universitaria y la incidencia que tuvieron las normativas que rigen la vida institucional de la Universidad Nacional de Asunción. El presente artículo corresponde a esta primera etapa.

El periodo de análisis abarca la década del 9o, coincidente con el inicio de la transición democrática en el país, luego de la caída de la dictadura.

El relevamiento de la información se realizó a través de una matriz en el que se recabóloscambioslegalesregistradosapartir de la ley máxima del país, y posteriormente rescatando los instrumentos de derechos humanos ratificados y los compromisos internacionales asumidos por el Paraguay, que marcan un estándar sobre el derecho humano a la educación universitaria.

En una segunda fase de este trabajo, se prevé realizar entrevistas a actores claves para analizar la incidencia de los marcos legales en la política de la UNA desde la perspectiva de actores de la comunidad educativa (estudiantes, docentes y egresados/as).

\section{Resultados y Discusión}

\subsection{Educación universitaria y el marco de los derechos humanos}

Laeducación en generalyla educación superior en particular, no pueden ser consideradas como un mero servicio o una mercancía, sino como una obligación que tiene el Estado de respetarla, asegurarla, protegerla y promoverla. Específicamente, la educación superior debe ser accesible y de calidad para todos y debe dejar de ser el último escalón de la educación formal, para constituirse en la autorización para la educación a lo largo de la vida (ANEAES, s.f.).

Los instrumentos de derecho internacional y nacional establecen garantías jurídicas en los términos de lo que plantea Courtis (2011), y se construye un nuevo concepto de calidad educativa congruente con los derechos humanos.

La propia Declaración Universal de los Derechos Humanos, imprime un marco general del derecho a la educación y, en su artículo 26 señala que:

1. Toda persona tiene derecho a la educación. La instrucción técnica y profesional habrá de ser generalizada; el acceso a los estudios superiores será igual para todos, en función de los méritos respectivos.

Este mismo marco agrega, en su inc 2) La educación tendrá por objeto el pleno desarrollo de la personalidad humana y el fortalecimiento del respeto a los derechos humanos y a las libertades fundamentales; 
favorecerá la comprensión, la tolerancia y la amistad entre todas las naciones y todos los grupos étnicos o religiosos.

Paraguay ha ratificado instrumentos de derechos humanos relacionados al derecho a la educación, entre ellos, el Protocolo Adicional a la Convención Americana sobre Derechos Humanos en Materia de Derechos Económicos, Sociales y Culturales (Protocolo de San Salvador) ${ }^{1}$ a nivel del Sistema Interamericano, y el Pacto de Derechos económicos, sociales y culturales $^{2}$ a nivel del Sistema Universal, en cuyo marco el Estado paraguayo ha asumido obligaciones.

El Pacto de Derechos Económicos, Sociales y Culturales, ratificado por Ley 4/92 en su artículo 13 consagra que:

1. Los Estados Partes en el presente Pacto reconocen el derecho de toda persona a la educación. Convienen en que la educación debe orientarse hacia el pleno desarrollo de la personalidad humana y del sentido de su dignidad, y debe fortalecer el respeto por los derechos humanos y las libertades fundamentales"

2. En el inc. c) agrega que "la enseñanza superior debe hacerse igualmente accesible a todos, sobre la base de la capacidad de cada uno, por cuantos medios sean apropiados, yen particular por la implantación progresiva de la enseñanza gratuita

El Comité de Derechos Económicos, Sociales y Culturales, organismo que interpreta y vigila el cumplimiento del Pacto Internacional de Derechos Económicos, Sociales y Culturales, en su Observación General $\mathrm{N}^{\circ} 13$ sobre el derecho a la educación, afirmó que éste "es el principal medio que permite a adultos y menores

1 Suscripto por Ley 1040/ 97.

2 Ratificado por Ley 4/92. marginados económica y socialmente salir de la pobreza y participar plenamente en sus comunidades".

El Pacto de Derechos Económicos sociales y Culturales en el sistema universal dispone que, "La enseñanza superior comprende los elementos de disponibilidad, accesibilidad, aceptabilidad y adaptabilidad, que son comunes a la enseñanza en todas sus formas y en todos los niveles"3. Expresamente contempla que cuando se trata de la educación superior se pide a los Estados Partes garantizar la no discriminación y que se implanten gradualmente la enseñanza superior gratuita. Por otro lado, en el sistema interamericano, Paraguay ha ratificado el Protocolo de San Salvador y en su artículo 13 inc. b) establece que "La enseñanza superior debe hacerse igualmente accesible a todos, sobre la base de la capacidad de cada uno, por cuantos medios sean apropiados y en particular, por la implantación progresiva de la enseñanza gratuita;".

Paraguay también adquirió compromisos internacionales con relación a la educación superior. En el año 2009, adhirió a la Declaración Mundial sobre Educación Superior que establece la educación como un bien público y que el acceso a ella debe ser igual para todos. Dicha Declaración también contempla que la educación superior es un instrumento para la eficaz lucha contra la pobreza, el desarrollo sostenible y sostenido, en fin para el progreso personal y social.

\subsection{Evolución de inclusión del derecho a la educación en el marco legal nacional}

El derecho a la educación superior ha tenido una importante evolución con la inclusión de estándares de derechos humanos. Esta evolución se refleja a través de los cambios en los marcos legales, sin embargo, se encuentran pendientes

3 Recomendación General No 13 párr. 17. 
las políticas públicas y los mecanismos institucionales que garanticen su cumplimiento.

Tal como señala Torres (2006)

la educación es tanto un derecho humano como un medio vital para promover la paz y el respeto por los derechos humanos y las libertades fundamentales. A fin de que se realice su potencial para contribuir a construir un mundo más pacífico, la educación debe ser universal y accesible de manera igualitaria para todos y todas. (p. 1)

La inclusión del derecho a la educación superior universitaria en el marco constitucional fue producto del propio proceso de democratización y vigencia del Estado de derecho en Paraguay.

Durante varias décadas, la Constitución de 1870 , de inspiración liberal, no incluyó el derecho a la educación superior y universitaria. Más adelante con la reforma constitucional y la vigencia de la Constitución de 1940, durante la Presidencia de José Félix Estigarribia, el Poder Ejecutivo podía controlar a la prensa, reprimir grupos privados, suspender las libertades individuales, y tomar medidas excepcionales para el bien del Estado, esto demuestra la inspiración dictatorial de dicha constitución.

En la de 1967, se incluye la mención de que el gobierno fomentará la educación superior universitaria. Adicionalmente en esta última, se incluye que además del fomento, el sostenimiento y la creación de becas para la formación superior. La Constitución de 1967 reglamentaría el alcance la autonomía universitaria.

Ya en el proceso de democratización iniciado en el país, luego de la caída de la dictadura, la reforma Constitucional del año 1992, trajo consigo cambios significativos en la inclusión de estándares de derechos humanos en torno a la educación universitaria. Por un lado incluyó el derecho a la educación de manera específica, contempló como función del Estado fomentar la educación superior y universitaria, y consagró la autonomía de las universidades, incluida la libertad de enseñanza y la cátedra.

Igualmente, en esta Constitución, las Universidades están facultadas a establecer en sus estatutos sus formas de gobierno y a elaborar sus planes de estudio de acuerdo con la política educativa y los planes de desarrollo nacional; incluye además como derecho, el otorgamiento de becas para estudiantes de escasos recursos económicos.

El marco normativo nacional sirvió de base para un progresivo desarrollo de normativas sobre la educación superior y universitaria y particularmente tuvo efectos en la modificación normativa interna en la UNA.

Desde la apertura democrática de 1989, se ha producido un fenómeno masivo de creación de universidades. De dos universidades existentes hasta 1990 se pasó a 54 en el año 2013, de las cuales 8 son públicas (sólo el 10\%) y 46 privadas (Gómez, 2016), es decir se tuvo un crecimiento total del $85 \%$.

Tal como señala Rivarola (2008, p. 560), el crecimiento del número de universidades se reflejó igualmente en la acelerada expansión de la matrícula universitaria. En el año 200o, la matrícula de las universidades públicas ascendía a 27374 estudiantes $(45,7 \%)$, en tanto que la de las universidades privadas alcanzó un total de 32503 alumnos que representaron el $54,3 \%$.

Desde la Ley 136/93 -hoy derogada-, las universidades fueron declaradas de interés social y se les liberó de todo impuesto fiscal o municipal; en caso de recibir legados serían exonerados de impuestos.

Surge la pregunta en este contexto, ¿en qué medida el crecimiento del número de universidades privadas no explica en parte por la liberación de impuestos tanto fiscal o municipal, que fuera garantizado en el marco del artículo 18 de la Ley 136/93 y 
que luego fue mantenido en el marco de la Ley de Educación Superior?

Más adelante con la aprobación de la Ley 2529/o6 de Universidades se modifican los artículos 4, 5, 8 y 15 de la Ley 136 y entre otras cosas se incluye la facultad del Consejo de Universidades de elevar el dictamen para la creación de las Universidades al Congreso para la creación de una universidad, pero aclarando que este dictamen no sería vinculante en ningún caso. Con esta medida al parecer se pretendía contar con una opinión técnica para la aprobación de futuras Universidades y que no fuera sólo potestad del parlamento.

Esta ley reconoce que la educación superior es un bien público y, por ende, es un factor fundamental para el desarrollo del país, en democracia y con equidad; se reconoce y garantiza el derecho la educación superior como un derecho humano fundamental, sin embargo no se logra universalizar el acceso y permanencia para todos aquellos que quieran y estén en condiciones legales y académicas para cursarla.

\subsection{Autonomía universitaria y reformulación de la relación Estado - Universidad}

Existen diferentes visiones, incluso contrapropuestas, sobre el desarrollo institucional y el proceso de instauración de la autonomía de la UNA. Por un lado, Rivarola (2008, p. 560) señala que "el marco jurídico institucional tuvo su origen en la primera ley de universidades que se sancionó el 29 de junio de 1929 (Ley No. 1046) ${ }^{4}$. Este autor considera que esta ley, fue inspirada fuertemente por la reforma

\footnotetext{
4 "Recién el 21 de junio de 1929 pudo ser aprobada, con evidentes correcciones, la ley de reforma del Régimen Universitario. En el recordado documento se asientan las bases de una Universidad que ha dispuesto superar los lineamientos trazados en 1882 , cuando eran otras la época y de los intereses educacionales. Treinta y siete años más tarde se consideró oportuno proceder a su reemplazo" (UNA, 1990).
}

de Córdoba, estableció el principio de autonomía, la que fue reiterándose en la totalidad de las leyes promulgadas relativas a este estamento del sistema educativo nacional.

Sin embargo, según Rodríguez (2015, p. 565), la universidad pública no ha tenido autonomía del poder real, sino se constituyó en un aparato de las dictaduras. Conforme al artículo 5 tanto la Ley 356/56 como la Ley 1291/87 de Universidades, el Rector era nombrado por el Poder Ejecutivo de una terna propuesta por el Consejo Superior Universitario. La comunidad universitaria no tenía ninguna participación en la elección de sus autoridades.

En la Constitución Nacional de 1940, la Universidad Nacional de Asunción integraba el Consejo de Estado corporativo $^{5}$. El Consejo tenía entre sus funciones dictaminar sobre asuntos de orden financiero y económico y también podía dictaminar sobre los proyectos de decretos con fuerza de ley.

Launiversidadpúblicahistóricamente ha sido un espacio de expresión de la hegemonía del partido gobernante, a pesar de que tanto la Ley 356/56 como la Ley 1291/87 de Universidades, prohibía expresamente toda actividad política partidaria en los recintos de la Universidad; pero conforme a relatos de estudiantes universitarios, en la práctica hasta el año 1989, en la Universidad Nacional de Asunción se exigía estar afiliado al partido colorado, como requisito para el ingreso.

Como se señaló con anterioridad, la propia Constitución Nacional de 1967 en su artículo 91 contemplaba que las leyes regularían el alcance de la autonomía universitaria. Con la reforma constitucional del año 1992, se concretiza un cambio de fondo en torno la autonomía universitaria,

\footnotetext{
5 El artículo 62 de la carta de 1940 contemplaba que el Consejo de Estado, además de los Ministros del Poder Ejecutivo, representante del comercio, industrias agropecuarias, fuerzas armadas, Arzobispo, integraba el Rector de la Universidad Nacional.
} 
pues en el artículo 79, se establece que las universidades son autónomas y en ese entendido, se contempla que son estas las que deben establecer sus estatutos y formas de gobierno, así como elaborar sus planes de estudio de acuerdo con la política educativa y los planes de desarrollo nacional, poniéndose al día con la tendencia regional $^{6}$.

Conforme a esto último, se puede afirmar que, el derecho a la educación universitaria se institucionaliza a partir de la reforma constitucional de 1992 y se consolida con la ratificación por parte del Estado paraguayo de instrumentos de derecho internacional de derechos humanos que consagra la educación universitaria como un derecho humano fundamental.

Ya en el marco de su autonomía la asamblea Universitaria de la UNA, ha aprobado cinco Estatutos desde la década del noventa. La primera ha sido en el año 1996 y la última en el año 2014. Actualmente se está trabajando en la reforma de los Estatutos de la UNA que está en pleno desarrollo.

A pesar de las restricciones impuestas porlos marcos legalesquesefueronadoptando, la Universidad Nacional de Asunción en consonancia con la garantía constitucional de autonomía universitaria se define como "una institución de derecho público, de educación superior, autónoma, autárquica, con personería y patrimonio propio". La autonomía se define como "la plena capacidad de la Universidad Nacional de Asunción para dictar sus estatutos y normas que rijan, determinar sus órganos de gobierno, elegir autoridades, ejercer funciones de docencia e investigación y extensión".

6 Contienen cláusulas semejantes, por ejemplo, las constituciones de Bolivia (artículo 185), Brasil (artículo 207), Colombia (artículo 69), Costa Rica (artículo 84), Ecuador (artículo 75), El Salvador (artículo 61), Guatemala (artículos 82 y 85), México (artículo 3.VII), Nicaragua (artículo 125), Panamá (artículo 99), Perú (artículo 18) y Venezuela (artículo 109).
Dada la tendencia cada vez marcada de debilitar la autonomía de las Universidades, se fueron adoptando marcos legales que regulan la autonomía de las mismas. En un primer momento, con la Ley 1264, General de Educación sancionada en el año 1998, se reafirma que las universidades son autónomas y que establecerán sus propios estatutos y formas de gobierno, y se garantiza que elaborarán sus planes y programas, de acuerdo con la política educativa para contribuir con los planes de desarrollo nacional. Adiciona a este mandato la obligatoriedad de que las Universidades coordinen los planes y programas, en el marco de un único sistema educativo nacional de carácter público.

Más adelante, con la sanción de la Ley 4995/13 "De Educación Superior, que deroga la Ley 136/93 “de Universidades"7, corre riesgo la autonomía universitaria garantizada en el artículo 79 de la Constitución, cuando establece que el Consejo Nacional de Educación Superior (CONES) es el órgano responsable de proponer y coordinar las políticas y programas para la educación superior, y da potestad a este Consejo para intervenir las universidades e institutos superiores.

De igual forma, el artículo 92 de esta Ley contrario a las garantías del debido proceso contempla que "No procederá ningún tipo de recurso contra las decisiones y/o resoluciones referidas a la intervención, sean ellas emanadas del Ministerio de Educación y Cultura o del Consejo Nacional de Educación Superior". Esta disposición además de resultar inconstitucional, reproduce esquemas de prohibición propios de los sistemas autoritarios, que pretenden imponer sus decisiones en única instancia.

Cabe destacar que estudiantes universitarios nucleados en la Federación de Estudiantes por la Educación y otros

7 En el marco de esta Ley tanto las universidades públicas y privadas, eran creadas por ley previo dictamen favorable y fundado del Consejo de Universidades. 
movimientos estudiantiles, se opusieron de manera sistemática a la aprobación del proyecto de Ley de Educación Superior (LES), por considerarlo como riesgo de privatización de la educación universitaria, denunciando que la misma buscaba favorecer una perspectiva mercantilista de la educación en detrimento de la calidad educativa, científica y ética de la enseñanza universitaria. Esta ley fue aprobada a pesar de la fuerte resistencia estudiantil universitaria y de algunos sectores docentes que la consideraban un retroceso frente a las conquistas históricas obtenidas hasta la fecha.

Un aspecto clave en la relación Estado-Universidad es lo que guarda relación el financiamiento y el presupuesto destinado al derecho a la educación en general. Rivarola (2008, p. 565) señala que por más de un cuarto de siglo el régimen autoritario mantuvo el gasto educativo en sus más bajo niveles, sin sobrepasar nunca el $2 \%$ del Producto Interno Bruto (PIB). En contraste, uno de los hechos llamativos durante el ejercicio de los gobiernos que se sucedieron desde 1989, año en se inicia la transición democrática, ha sido el progresivo aumento de los recursos destinados a la educación, al punto de alcanzar alrededor del $20 \%$ del presupuesto nacional. (p. 565)

De acuerdo al artículo 76 de la LES, "Los recursos destinados a la educación superior de carácter público en el Presupuesto General de la Nación, no serán inferiores al 7\% (siete por ciento) del total asignado a Administración Central, excluidospréstamosydonaciones". También contempla que independientemente a este porcentaje, serán además destinados a la investigación un mínimo de $2 \%$ (dos por ciento) del Presupuesto General de la Nación.

\subsection{Desigualdad en la educación universitaria pública}

La universidad sigue siendo profundamente desigual, en el año 2011 "el $69 \%$ de la población de 19 a 25 años edad, grupo etario que corresponde a la educación superior de grado no asistió a ninguna institución educativa. Dicho promedio aumenta a $89 \%$ entre la población pobre extremo, $80 \%$ entre los pobres no extremos y baja a $63 \%$ entre los no pobres" (Molinier, 2012, p. 44).

De acuerdo al anuario estadístico (2015), la UNA en el periodo 2015 tuvo una población estudiantil de 47.517 universitarios de grado y 6.036 estudiantes de postgrado, nivel de Especializaciones, Maestrías y Doctorados, 9.052 Docentes y 5.641 Funcionarios Administrativos y de Servicios distribuidos para el servicio educacional en 79 carreras de grado. De la UNA egresan alrededor de cuatro mil profesionales por año.

Hoy se habla de la universidad de clase mundial, elitista, de calidad y que responda a los intereses del mercado (Ovelar, 2015). Esta política responde a una estrategia del Equipo Nacional de Estrategia País (ENEP), en el marco del Plan Nacional de Desarrollo 2030, en su eje 2 sobre crecimiento económico inclusivo, el de contar con al menos una universidad paraguaya ubicada entre las primeras 400 a nivel mundial y quintuplicar el número de patentes de invenciones nacionales.

La universidad pública afronta hoy un gran desafío cuando se reivindica como relación social privilegiada la generación de renta por parte de empresas privadas, en cuyo contexto el rol de la universidad pasa a ser el de un mero colaborador de la empresa.

Mientras se plantea impulsar la creación de una universidad de élite, el acceso y la permanencia en la educación universitaria representan marcados obstáculos para el cumplimiento del derecho a la educación superior. El Informe socioeconómico de ingresantes 
de la UNA (2014, p. 7), revela que siete de cada diez encuestados pertenecen al nivel socioeconómico medio bajo $\mathrm{y}$ bajo. Específicamente predominan los ingresantes del nivel socioeconómico medio bajo $(53,20 \%)$, medio $(25,30 \%)$, $\mathrm{y}$ en menor proporción los niveles bajo $(18,57 \%)$ y medio alto y alto $(2,94 \%)$. En general, provienen de colegios públicos, no trabajan y piensan solventar sus estudios universitarios con ayuda familiar, cuyos ingresos alcanzan hasta dos salarios mínimos.

El informe socioeconómico citado más arriba (2015, p. 13) se revela que el Instituto "Dr. Andrés Barbero" cuenta con el mayor porcentaje $(40,40 \%)$ de ingresantes con nivel socioeconómico bajo, seguido por el Instituto de Trabajo Social $(35,56 \%)$. Cabe destacar que solo el $2,94 \%$ de los estudiantes pertenece al nivel socioeconómico medio alto y alto.

Las formas de solventar los estudios de los ingresantes, según el informe socio económico de la UNA (2015, p. 18), el 62,8\% lo realiza con ayuda familiar, seguida de quienes lo realizan por cuenta propia $(24,8 \%)$. En un porcentaje mucho menor están los que reciben becas o exoneraciones $(12,0 \%)$.

Aunque la LES contempla como derecho de los estudiantes en su artículo 47, inc. d) el "Obtener becas, créditos y otras formas de apoyo económico y social que garanticen la igualdad de oportunidades y de condiciones, particularmente para el acceso y permanencia en los estudios de grado y postgrado, conforme a las normas que reglamenten la materia", en el estudio de la UNA sobre Experiencias de deserción en Universidades (2012), se señala que las principales causas de abandono manifestados por orden de prioridad son entre otros, el bajo nivel académico previo al ingreso y problemas económicos del grupo familiar.

\subsection{Participación estudiantil y demandas por la autonomía y democratización de la UNA}

La relación de la Universidad con los estudiantes y la comunidad académica, producto de la dictadura se ha caracterizado por algunas prácticas de represión hacia las y los estudiantes en la UNA. Un ejemplo de ello es que a la luz de la Ley 1048/año, el Consejo Superior Universitario dispuso que a los fines de velar por la efectividad del orden y disciplina en la jurisdicción de la UNA, así como para asegurar el mantenimiento de la autoridad y jerarquía superiores de la tribuna universitaria, "Queda prohibido a los alumnos de todas las Facultades a celebrar reuniones sin permiso de las autoridades universitarias" (UNA, 1947, p. 41).

Ya en el contexto democratización de las Universidades, la LES en su artículo 47 inc. c) contempla como derecho de los estudiantes de educación superior el de "Asociarse libremente en centros de estudiantes, elegir a sus representantes y participar en el gobierno y en la vida de la institución, conforme a los estatutos, lo que establece la presente Ley y demás disposiciones legales que rigen la materia”.

Sin embargo, a pesar de estos disposiciones, las prácticas antidemocráticas siguieron en algunas unidades académicas, como el ejemplo que señala Krug (2013, pp. 259-260), la Decana de Filosofía en el año 2013 presentó denuncia al Ministerio Público donde sindicaba a 52 estudiantes y un docente de haber cometido hechos punibles de coacción, coacción grave y privación de libertad. La denuncia se basó en una serie de movilizaciones que tuvieron su punto de origen en un paro estudiantil de la carrera de Psicología - especialidad Clínica por la destitución injusta y arbitraria de una docente.

La lucha por la autonomía, la educación gratuita y la democratización de la UNA tiene sus antecedentes históricos. La denominada "primavera universitaria democrática” movilizada por estudiantes en 
setiembre del 2015, fue una rebelión contra un modeloautoritario, excluyenteycorrupto dentro de la UNA. Esta movilización exigió la transparencia, la calidad educativa, una gobernanza democrática y la construcción de una universidad comprometida con el pueblo. El estudiantado recuperó su fuerza y su palabra, su horizonte de futuro, la esperanza y los recuerdos que parecían haberse perdido. Es que parece una paradoja, que los estudiantes no hagan nada, aparentemente nada, o hacen la historia (Rodríguez, 2015).

Esta movilización estudiantil, trajo como resultado la renuncia y procesamiento del Rector Prof. Dr. Froilán Enrique Peralta; luego se fueron sumando las demandas de la rendición de cuentas del uso de los recursos públicos, y la petición de saneamiento administrativo y financiero dentro de la UNA.

Ante la situación de crisis se dieron amenazas de intervención del CONES para "restablecer el funcionamiento organizacional". Como respuesta a esto según el Plan entregado al CONES, la UNA se comprometió en conformar un Equipo Interestamental responsable de la conducción del proceso de elaboración y redacción del nuevo estatuto estableciendo mecanismos expresos de consultas, aportes y socialización. La amenaza de intervención puso de relieve la falta de autonomía de las universidades que está legalmente reconocida en la Ley de Educación Superior.

\section{Conclusiones}

El trabajo aporta una aproximación sobre las reformas legales y la inclusión de estándares de derechos humanos en la educación superior en la Universidad pública en Paraguay.

La Universidad Nacional de Asunción (UNA), como primera universidad pública creada en 1889, con el lema "Vitam impendere vero" que en latín significa "Consagrar la vida a la búsqueda de la verdad", en su marco legal vigente aún presenta grandes brechas para la inclusión de estándares de derechos humanos.
Aunque formalmente las leyes contemplaban la autonomía universitaria, las mismas disponían contradictoriamente que el Rector debía ser electo por el Poder Ejecutivo. Recién en el contexto de la transición democrática, se garantiza la autonomía de las universidades con la Constitución Nacional y otras normativas. Sin embargo, a pesar de los avances registrados en la educación universitaria persisten brechas para el acceso y permanencia de los sectores empobrecidos en las universidades públicas.

Los actores estudiantiles históricamente han demandado autonomía y una educación universitaria pública y gratuita. En la década del noventa en Paraguay se impulsó y concretó la reforma de la educación primaria y secundaria, se crearon las condiciones para la expansión y crecimiento de las universidades privadas.

Es preciso la inclusión de principios de igualdad y no discriminación, las garantías de una educación universitaria pública gratuita y de calidad conforme a las obligaciones internacionales. También se requiere fortalecer la vinculación social de la UNA, y derribar los muros y obstáculos para la enseñanza basada en privilegios y convertirla en espacios de producción y difusión de los conocimientos socialmente necesarios para comprender y trasformar la sociedad paraguaya.

\section{Referencias Bibliográficas}

ANEAES. La creación de la Agencia Nacional de Evaluación y Acreditación de la Educación Superior. Recuperado de http://www.aneaes.gov.py/aneaes/ index.php/ct-menu-item-2

Caballero, J. (2014). La experiencia de intervención internacional para la equidad en la educación superior de la Red Riaipe entre instituciones de educación superior de Paraguay. AL y la UE. Revista Paraguaya de Educación, 4, 87-100.

Courtis, C. (2011). Políticas sociales, programas sociales, derechos sociales. 
Ideas para una construcción garantista. In V. Abramovich \& L. Pautassi L. (Ed.), La medición de derechos en las políticas sociales. Buenos Aires.

Gómez, G. (2016). Políticas de Educación Superior del MEC. Fortalecimiento de la educación superior. Formación de educadores de calidad. Presentado ante el REUNA. Asunción.

Krug, F. (2013). La imperiosa necesidad de democratizar la universidad. In Foro por el Derecho a la Educación (pp. 257263). Asunción: CODEHUPY.

Ley 4/1992. Que aprueba la adhesion de la República del Paraguay al Pacto Internacional de Derechos Económicos, Sociales y Culturales, adoptado durante el XXI Periodo de Sesiones de la Asamblea General de la Organización de las Naciones Unidas, en la Cuidad de Nueva York, el 16 de diciembre de 1966. 24 de marzo de 1992. Recuperado de http://www. cej.org.py/desc/desc/Adriana\%20 desc/tierra/Intrumentos \% 20 internacionales/Ley\%204-1992\%20 de\%2oadhesi $\% \mathrm{C}_{3} \% \mathrm{~B}_{3} \mathrm{n} \% 2 \mathrm{oal} \% 2 \mathrm{o}$ PIDESC.doc

Ley 1040/1997. Que aprueba el Protocolo de San Salvador, del 16 de abril de 1997. 20 de marzo de 1997. Recuperado de http://www.iadb.org/research/ legislacionindigena/leyn/docs/ParaPara-Ley-1040-97-Protocolo-SanSalvador-.doc

Ley 136/1993. De Universidades. 29 de marzo de 1993. Recuperado de http://www. cej.org.py/games/Leyes_por_Materia juridica/EDUCACION/LEY\%20136.pdf

Ley 1264/1998. Ley General de Educación. 26 de mayo de 1998. Recuperado de http://www.pol.una.py/sites/ default/files/files/reglamentos/ Ley1264GeneralDeEducacion.pdf

Ley 4995/2013. Ley de educación superior. 2 de agosto de 2013. Recuperado de http:// www.sansebastian.edu.py/uassweb/ file/Ley_4995_De_Educacion_ Superior.pdf
López, F. (2008). Tendencias de la educación superior en el mundo y en América Latina y el Caribe. Recuperado de http://www.ses.unam.mx/curso2014/ pdf/LopezSegrera20o8.pdf

Molinier, L. (2012). Caracterización general del sistema educativo. El sistema educativo gratuito y la educación superior. Presentado en el Foro por el derecho a la educación. Asunción.

Ovelar, W. (2016, enero 5). El Paraguay y su futura universidad de clase mundial (I). $A B C$ Color. Recuperado de http://www. abc.com.py/edicion-impresa/opinion/ el-paraguay-y-su-futura-universidadde-clase-mundial-i-1441593.html

Rivarola, D. (2003). La educación superior universitaria en Paraguay. Asunción: Ministerio de Educación y Cultura. IESLAC.CONEC.

Rivarola, D. (2008). La universidad paraguaya, hoy. Avaliação: revista da Avaliação da Educação Superior, 13(2), 533-578.

Rodríguez, J. C. (2015). La primavera estudiantil. In Informe de Derechos Humanos (pp. 565-568). Asunción.

Tomasevski, K. (2004). Los derechos económicos, sociales y culturales. El derecho a la educación. Informe de la relatora especial. E/CN.4/2004/45/ Add.2. Naciones Unidas.

Torres, R. M. (2006). Derecho a la educación es mucho más que acceso de niños y niñas a la escuela. In $X$ Congreso Nacional de Educación Comparada "El derecho a la educación en un mundo globalizado". Sociedad Española de Educación Comparada, San Sebastián, España (pp. 6-8).

Tünnermann, C. (2008). La Reforma de Córdoba. Vientre fecundo de la transformación universitaria en La reforma universitaria: desafíos $y$ perspectivas noventa años después. Buenos Aires: CLACSO. Recuperado de http://biblioteca.clacso.edu.ar/ar/libros/ grupos/reforAboit/reformaAboites.pdf 
UNA. (1947). Guía de la Universidad Nacional de Asunción. Asunción: UNA.

UNA. (1990). Historia de la Universidad Nacional de Asunción (1889-1989). Volumen I (1889-1915). Asunción: UNA.

UNA (2012). Experiencias sobre deserción en Universidades del Paraguay. Recuperado de http://www.una.py/ images/stories/Universidad/Estadistica/ ExperienciasdesercinParaguay.pdf

UNA. (2014). Informe socioeconómico de ingresantes en el año 2014. Recuperado de http://www.una.py/images/ stories/Universidad/Estadistica/ InformeSocioecom/1. Informe Socioeconomico_2015.pdf

UNA. (2015). Anuario estadístico de la UNA. Recuperado de http://www. una.py/images/stories/Universidad/ Estadistica/Anuario/Anuario_ Estadistico_2015.pdf
UNESCO. (2007). Educación de Calidad para Todos. Un Asunto de Derechos Humanos. Documento de discusión sobre políticas educativas en el marco de la II Reunión Intergubernamental del Proyecto Regional de Educación para América Latina y el Caribe (EPT/ PRELAC). Buenos Aires. Recuperado de http://unesdoc.unesco.org/ images/o015/001502/150272s.pdf

UNESCO. (2015). Educación 2030: Hacia una educación inclusiva y equitativa de calidad y un aprendizaje a lo largo de la vida para todos. Recuperado de http://es.unesco.org/world-educationforum-2015/about-forum/declaracionde-incheon

Zarate, E., Cornejo, R., Carhuapoma, M. \& Arca, J. (2008). Retos universitarios: reforma de la reforma. Anales de la Facultad de Medicina, 69(2), 140-145. 